\title{
A sparsity-inducing optimization based algorithm for planar patches extraction from noisy point-cloud data
}

\author{
Guangcong Zhang*, Patricio A. Vela, Peter Karasev \\ School of Electrical and Computer Engineering, Georgia Institute of Technology, Atlanta, GA, USA, 30332
}

$\&$

Ioannis Brilakis

Department of Engineering, University of Cambridge BC2-07, Trumptington Street, Cambridge, CB2 1PZ, UK.

\begin{abstract}
Currently, much of the manual labor needed to generate as-built Building Information Models (BIMs) of existing facilities is spent converting raw Point Cloud Datasets (PCDs) to BIMs descriptions. Automating the $P C D$ conversion process can drastically reduce the cost of generating as-built BIMs. Due to the widespread existence of planar structures in civil infrastructures, detecting and extracting planar patches from raw PCDs is a fundamental step in the conversion pipeline from PCDs to BIMs. However, existing methods cannot effectively address both automatically detecting and extracting planar patches from infrastructure PCDs. The existing methods cannot resolve the problem due to the large scale and model complexity of civil infrastructure, or due to the requirements of extra constraints or known information. To address the problem, this paper presents a novel framework for automatically detecting and extracting planar patches from large-scale and noisy raw PCDs. The proposed method automatically detects planar structures, estimates the parametric plane models, and determines the boundaries of the planar patches. The first step recovers existing linear dependence relationships amongst points in the PCD by solving a group-sparsity inducing optimization problem. Next, a spectral clustering procedure based on the recovered linear dependence relationships segments the PCD. Then, for each segmented group, model parameters of the extracted planes are estimated via Singular Value Decomposition (SVD) and Maximum Likelihood Estimation Sample Consensus (MLESAC). Finally, the $\alpha$-shape algorithm detects the boundaries of planar structures based on a projection of the data to the planar model. The proposed approach is evaluated comprehensively by experiments on two types of PCDs from real-world infrastructures, one
\end{abstract}

captured directly by laser scanners and the other reconstructed from video using structure-from-motion techniques. In order to evaluate the performance comprehensively, five evaluation metrics are proposed which measure different aspects of performance. Experimental results reveal that the proposed method outperforms the existing methods, in the sense that the method automatically and accurately extracts planar patches from large-scaled raw PCDs without any extra constraints nor user assistance.

\section{INTRODUCTION}

Traditional Building Information Models (BIMs) represent the conditions under which a facility is designed. However, the reality of the facility's construction can differ from the nominal design. Furthermore, changes in facility's conditions may happen during the life span of the facility. Hence, generating as-built BIMs, which aim to capture the as-built conditions of facilities, have been a recent topic of interest in the literature (Huber et al., 2011). Generating asbuilt BIMs usually consists of two phases (Goedert et al., 2005): (1) data collection; and (2) objects identification, extraction, and modeling. Current developments in technologies and techniques for remote spatial sensing, e.g. high density LiDAR (Deshpande 2013), image-based 3D reconstruction (Seitz et al., 2006; Furukawa et al., 2010) and video-based structure-from-motion (Zhang et al, 2012; Davison et al., 2007; Pollefeys et al., 2008), have largely simplified and facilitated the data collection process such that generating dense point clouds with color information of target objects is quickly becoming standard. Nevertheless, fully and simply automating the phase of objects identification, extraction and modeling remains an open problem. 


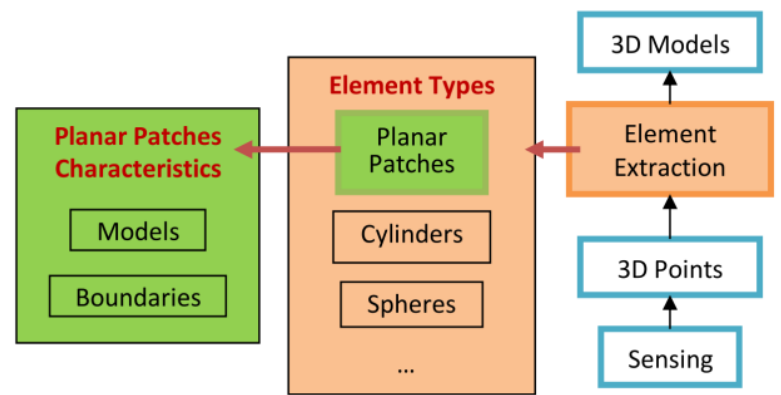

Figure 1: Role of planar patches extraction in the automatic conversion from raw PCDs to 3D models

The difficulty of automating "objects identification, extraction, and modeling" lies in that a raw Point Cloud Dataset (PCD) provides only Cartesian measurements and no knowledge of the elements contained therein (i.e. which parts of the PCD belong to which entities? which parts are from which geometric shapes?), nor does it immediately provide any other as-built information (i.e. changes in building conditions, etc.). To automate the process of generating as-built BIMs, recognition of infrastructure elements needs to be automated during the conversion from raw PCDs to 3D models, as shown in Figure 1.

Because of the widespread existence of planar structures in civil infrastructures, automatic extraction and modeling of planar structures are fundamental steps in automating the conversion process. Automatic extraction and modeling of 3D planar structure methods requires detecting planes, estimating planar model parameters, and determining planar patch boundaries. Exisiting software, e.g., AutoCAD, Paraview, Kubit-Pointcloud, is not able to achieve all of these steps fully automatically. Accordingly, this paper focuses on developing algorithms to achieve these three necessary steps.

The main motivation for this work is to develop a global, complete and accurate algorithm for planar patch modeling from PCDs, which will be further used in the generating a building information model. However, this algorithm is also valuable to many other communities which require environment modeling, including robot perception of 3D environments, $\mathrm{CAD}$ for mechanical engineering, inverse engineering, shape modeling in computer graphics community, 3D reconstruction in computer vision community, etc.

\section{STATE OF THE RESEARCH}

Techniques for 3D surface modeling from point cloud data can be found in computer graphics literature. Most of the algorithms are based on building meshes from point clouds with different explicit representations of surfaces. The problem of representing surfaces was partially addressed by Farin, G., et al., who proposed triangular meshes (Farin, 1992; Farin, 1996). Although modeling through surfaces meshes gives an explicit description of the object's surfaces, it fails to give information about the parameters of the surfaces' geometric models and thus they are not suitable to be used in generating as-built BIMs.

Different from mesh-based 3D surface reconstruction, model-based surfaces reconstruction requires the detection and extraction of embedded surface models in PCDs. Many techniques for shape models extraction are based on Random Sample Consensus (RANSAC) algorithm (Schnabel et al., 2007). In civil engineering applications, Tarsha-Kurdi (Tarsha-Kurdi et al., 2008) applied RANSAC to building roof detection. Unfortunately, fully automatic RANSAC based methods usually have very high computational complexity when applied to large-scale, complex PCDs with multiple embedded surface models. To overcome the high complexity of RANSAC, Bosché (Bosché, 2012) presented a semi-automatic RANSAC based method requiring manual plane selection.

Another approach for extracting planar models from PCDs utilizes the Hough transform (Tarsha-Kurdi et al., 2008). Landes (Landes et al., 2007) compared 3D Hough transform based algorithms to RANSAC based algorithms for automatic detection of planes from PCDs, and found that RANSAC is better than the 3D Hough-transform in terms of speed and percentage of successful detections. To improve the traditional Hough transform based method, Okorn and Huber (Okorn et al., 2010, Huber et al., 2011) combined it with 2D image histograms to automatically model as-built floor plans. However, the approach is not able to achieve very high accuracy because of the voxelization step used in generating the 2D histograms.Also, it requires proper alignment of the PCD wi the coordinate axes.

Other planar surfaces extraction methods proposed in the recent years include the following. The plane-sweep search algorithm presented in (Budroni et al., 2009), which utilizes the distribution of the $3 \mathrm{D}$ points along different directions to recognize the parts which contain planes and then further extract the planes within each part. The region-growing methods proposed in (Hähnel et al., 2003), which extracts planes by first picking a seed point and then growing the planar region from this point if criteria based on the normal deviation and mean square error are satisfied. Adan and Huber (Adan et al., 2011; Huber et al., 2011) also presented a modified region growing method on a voxelized PCD which connects nearby points with similar surface normals and that are well described by a planar model when aggregated. Another modified region-growing method is presented in (Dorninger et al., 2008), which is optimized for airborne laser scanned point clouds. This method is initialized by seed clusters in the feature space defined by local regression planes. (Nevado et al., 2004) also utilized a region growing method but with the normal computed from adaptive-radius neighboring regions. In a more recent study (S.B. Walsh et al., 2013), a modified seeded regiongrowing method combined with sharp features is employed 


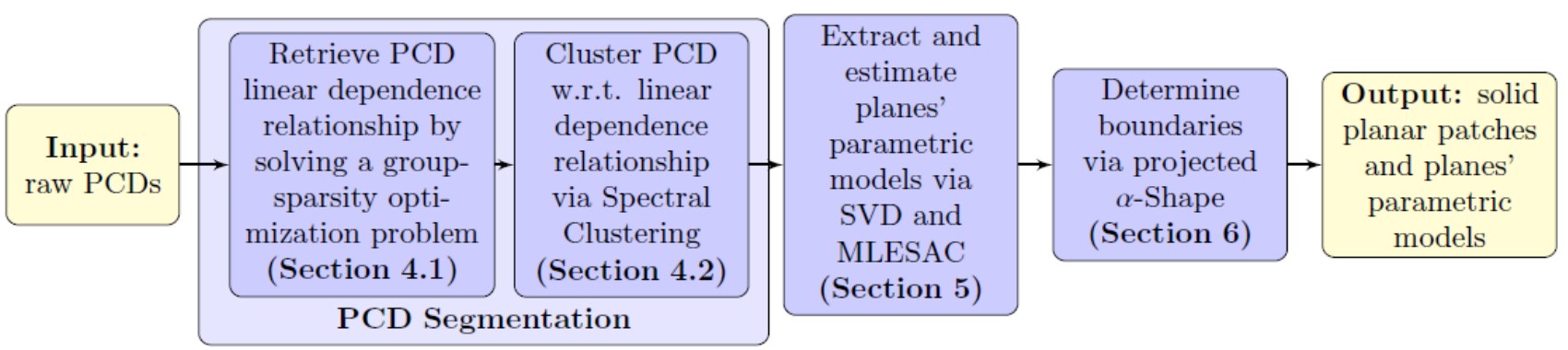

Figure 2 The proposed methodology

for segmenting the PCD. The paper (Vosselman, G., 2009) summarizes the major methods for point cloud processing, in which several segmentation methods are discussed, including RANSAC, Hough transform, and surface growing. Different from the above methods, other methods include: machine-learning based methods using the Expectation-Maximization (EM) algorithm (Thrun et al., 2004) or hierarchical EM (Triebel et al., 2005), and a geometry-based method using clustering with co-normality and co-planarity metrics (Stamos et al., 2000), etc. However, the existing methods discussed above do not provide a complete and global solution to fulfill the requirements of automatically detecting planes, estimating plane models and determining the patches boundaries without requiring the number the patches as input. For example, plane-sweeping algorithms focus on plane detection, region growing methods focus on segmentation of the PCD, RANSAC based methods do detection and estimation but do not extract the boundaries and the RANSAC family are intrinsically randomized which cannot provide a complete solution.

\section{PROBLEM STATEMENT AND OBJECTIVES}

The objective of this work is to develop an algorithm which takes raw point cloud data as input, and outputs a collection of planar patch models. The planar patch model description consists of the plane model parameters and the patch boundary. The planar patches found in the point cloud serve as a substitute structure for visualizing the infrastructure modeled by the point cloud, and serve as an intermediate representation in the PCD to BIM conversion pipeline. More specifically, the algorithm should admit as input a civil infrastructure PCD, which is typically large scale, embedded with multiple shape components, and corrupted by noise. The algorithm should admit PCDs generated from different sources, e.g., videos, photos, range images (LiDAR), laser scanning, etc. To be agnostic to the data source, the algorithm will not exploit additional sensor-specific data that may be available (e.g., topology information available from range image cameras). In addition, the algorithm should not require advanced knowledge of the number of planar patches nor any assumptions concerning their geometry (such as alignment to specific axes). The output should include the parametric models and the boundaries of the planar patches.

The literature and the commercial software to date, do not provide a method that can fully automatically detect and extract planar patches without requiring the quantity of planar patches as input, then estimate plane models and determine the patch boundary with high accuracy, although some software is able to extract planar patches with user interaction. The existing methods cannot resolve the stated problem due to one or more of the following reasons: the model extraction will be incomplete, civil infrastructure consists of multiple joined models to segment and estimate, the algorithms rely on specific geometric properties (such as the alignment of planar regions to specific coordinate axes), or the algorithms require user-provided information (such as the quantity of planar patches), etc. The method proposed to resolve the stated problem, and detailed in subsequent sections, is summarized in Figure 2. The first step introduces a segmentation algorithm for PCDs utilizing unsupervised subspace learning techniques (Vidal, 2011) modified for the case of PCDs (Section 4). This step retrieves the embedded linear dependence relationship between the points in $\mathbb{R}^{3}$ space and then segments the PCDs w.r.t. this relationship, such that within each segmented group there is at most one embedded linear subspace. Next, for each segmented part, the application of Maximum Likelihood Estimation Sample Consensus (MLESAC) (Torr et al., 2000), in conjunction with Singular Value Decomposition (SVD) based plane model estimation, robustly estimates and verifies the plane models (Section 5). The points in the PCD are evaluated against the estimated models to correct the segmentation. For the last step, described in Section 6, the boundaries of the planar patches are determined by extracting the boundary points of the concave hull of each extracted plane, by utilizing the $\alpha$ shape algorithm (Akkiraju et al., 1995).

Sections 4-6 provide a formal description of each algorithm. Furthermore, a synthetic example is processed in each section to illustrate the outputs associated to each step of the complete algorithm. Section 7 provides analysis of 
the memory and computation complexities, evaluation metrics, and evaluation of the experiment on the synthetic example. The proposed algorithm is comprehensively evaluated using data from two real-world infrastructure PCDs in Section 8. The two real-world datasets used were captured using different sensing methods: one is reconstructed from video using structure-from-motion techniques and the other is captured directly using a professional laser scanner (Leica Scan Station C10). The last section of the paper provides a conclusion of our work.

A previous version of our algorithm was presented in (Zhang et al., 2012). However, the algorithm in this paper has various improvements over the last one and this paper provides more technical details of the algorithm.

\section{POINT CLOUDS SEGMENTATION BY CLUSTERING SPARSE LINEAR SUBSPACES}

The proposed algorithm begins with the segmentation of a PCD according to the embedded linear subspaces of $\mathbb{R}^{3}$. The reason to segment PCDs as a first step is that robust parametric estimation methods, such as RANSAC, are designed for datasets with one dominant underlying model. These methods are ineffective for datasets with multiple models, i.e., when more than one model can be fit from the dataset, or datasets without dominant models. Meanwhile, randomized estimation methods like RANSAC are of high computational complexity and are impractical when the cardinality of the point-set is large. Therefore, segmenting PCDs is necessary before extracting and estimating the plane models. However, segmentation of PCD may destroy the underlying planar structures embedded in the PCD. Hence, the segmentation step should preserve the underlying planar structures.

Segmenting PCDs while preserving underlying models is a subspace clustering problem (also known as unsupervised subspace learning). Given a point-set $\left\{\boldsymbol{y}_{\boldsymbol{i}} \in \mathbb{R}^{D}\right\}_{i=1}^{N}$ containing a union of $n$ linear or affine subspaces in $\mathbb{R}^{D}$, let $\left\{\boldsymbol{S}_{l}\right\}_{l=1}^{n}$ be an arrangement of the $n$ subspaces of dimensions $\left\{d_{l}\right\}_{l=1}^{n}$. The subspaces can be expressed as:

$$
\boldsymbol{S}_{\boldsymbol{l}}=\left\{\boldsymbol{y} \in \mathbb{R}^{D}: \boldsymbol{y}=\boldsymbol{\mu}_{\boldsymbol{l}}+\boldsymbol{U}_{\boldsymbol{l}} \boldsymbol{x}\right\}, l=1, \ldots, n
$$

where $\boldsymbol{\mu}_{\boldsymbol{l}} \in \mathbb{R}^{D}$ is an arbitrary point in subspace $\boldsymbol{S}_{\boldsymbol{l}}$ that can be chosen as $\boldsymbol{\mu}_{\boldsymbol{l}}=0$ for linear subspaces, $\boldsymbol{U}_{\boldsymbol{l}} \in \mathbb{R}^{D \times d_{l}}$ is a basis for subspace $\boldsymbol{S}_{\boldsymbol{l}}$, and $\boldsymbol{x} \in \mathbb{R}^{d_{l}}$ is a low-dimensional representation for point $y$. Subspace clustering refers to the process of finding the number of subspaces $n$, their dimensions $\left\{d_{l}\right\}_{l=1}^{n}$, the subspace bases $\left\{\boldsymbol{U}_{i}\right\}_{i=1}^{n}$, the points $\left\{\boldsymbol{\mu}_{i}\right\}_{i=1}^{n}$, and segmenting groups of points according to the subspaces. A number of subspace clustering algorithms have been proposed, broadly categorized into algebraic methods (Costeira et al., 1998; Vidal et al., 2005) iterative methods (Agarwal et al., 2004; Lu et al., 2006; Zhang et al., 2009), statistical methods (Ma et al., 2007; Rao et al., 2008), and spectral clustering-based methods (Zhang et al.,
2010; Elhamifar et al., 2009; Liu et al., 2010). In (Vidal, 2011), the author compared different subspace clustering methods, and reported that the Sparse Subspace Clustering (SSC) method proposed in (Elhamifar et al., 2009) had the best performance in terms of misclassification error. In (Soltanolkotabi et al., 2012), a geometric analysis of SSC is given proving that SSC can correctly cluster data points even when subspaces intersect. SSC is based on an $\ell_{1}$ optimized sparse representation. In the case of PCDs, due to the geometric nature of point clouds, $\ell_{2}$-norm penalties also capture the linear dependence relationship, and thus the linear dependence problem is formulated as an optimization problem to minimize the combined $\ell_{1}$ and $\ell_{2}$ penalties, denoted as group-sparsity optimization.

\subsection{Recovering PCD linear subspaces}

This section covers the recovery of linear subspaces in a PCD based on sparse optimization programming. Sparse optimization programming exploits the self-expressiveness property of the data, which presumes that each point of the

$$
\begin{array}{ll}
\underset{\boldsymbol{Z}}{\operatorname{minimize}} & \|\boldsymbol{Z}\|_{1}+\beta\|\boldsymbol{Y} \boldsymbol{Z}-\boldsymbol{Y}\|_{2} \\
\text { subject to } & \sum_{i} z_{i j}=1 ; j=1,2,3, \ldots \\
& \operatorname{diag}(\boldsymbol{Z})=\mathbf{0}, \quad \beta>0
\end{array}
$$

PCD can be expressed by linear combinations of other points from its underlying linear subspace.

\subsubsection{Sparse representations in subspaces}

Let the vector $x \in \mathbb{R}^{D}$ be representable by a basis $\boldsymbol{\psi}_{\boldsymbol{i}} \in \mathbb{R}^{D}, i=1,2, \ldots, N$ as follows:

$$
\boldsymbol{x}=\sum_{i=1}^{N} f_{i} \boldsymbol{\psi}_{\boldsymbol{i}}
$$

If $\boldsymbol{x}$ cannot be measured directly but its $k$ combination $\boldsymbol{y}$ can be measured, then

$$
\boldsymbol{y}=\sum_{j=1}^{k} \boldsymbol{\phi}_{i}\left(\sum_{i=1}^{N} f_{i} \boldsymbol{\psi}_{i}\right)=\boldsymbol{\Phi} \boldsymbol{\Psi} \cdot \boldsymbol{f}=\boldsymbol{\Omega} \boldsymbol{f},
$$

where $\boldsymbol{\Psi}=\left[\boldsymbol{\psi}_{1}, \boldsymbol{\psi}_{2}, \ldots, \boldsymbol{\psi}_{\boldsymbol{N}}\right]^{\top} \in \mathbb{R}^{D \times N}, \boldsymbol{f}=\left[f_{1}, f_{2}, \ldots, f_{N}\right]^{\top}$ $\in \mathbb{R}^{N}$ and $\boldsymbol{\Phi}=\left[\boldsymbol{\phi}_{1}, \boldsymbol{\phi}_{2}, \ldots, \boldsymbol{\phi}_{\boldsymbol{k}}\right]^{\top} \in \mathbb{R}^{k \times D}$. When $\boldsymbol{x}$ has a sparse representation in a basis $\boldsymbol{\psi}$, then a sparse representation is recoverable through the following convex optimization problem, also known as basis-pursuit $(B P)$ (Donoho et al., 2006) with the unknown $\boldsymbol{f}=\left[f_{1}, f_{2}, \ldots, f_{N}\right]$.

$$
\underset{f}{\operatorname{minimize}}\|\boldsymbol{f}\|_{1}, \text { subject to } \boldsymbol{y}=\boldsymbol{\Omega} \boldsymbol{f} \text {, }
$$

where $\|\cdot\|_{1}$ is the $\ell_{1}$-norm.

\subsubsection{Retrieving linear dependence relationships in PCD}

This section describes how to utilize the optimization of Equation (4) to generate a sparse representation of the PCD 
when it contains several planar subspaces and the data has measurement error. Let $\left\{\boldsymbol{S}_{i}\right\}_{i=1}^{m}$ be a union of $m$ independent linear subspaces of dimensions $\left\{d_{i}\right\}_{i=1}^{m}$ embedded in a $k$ dimensional space, and $\left\{\boldsymbol{y}_{i}\right\}_{i=1}^{N}$ be a collection of $N$ observations from the $k$ dimensional space, $\boldsymbol{y}_{\boldsymbol{i}} \in \mathbb{R}^{k}$. If $\boldsymbol{y}_{\boldsymbol{i}}$ belongs to subspace $\boldsymbol{S}_{\boldsymbol{j}}$, then $\boldsymbol{y}_{\boldsymbol{i}}$ is a linear combination of the other data points in $\left\{\boldsymbol{S}_{\boldsymbol{i}}\right\}_{i=1}^{m}$. To compensate for measurement error, the basis-pursuit problem of Equation (4) is modified to be a basis-pursuit denoising $(B P D N)$ problem:

$$
\begin{array}{ll}
\underset{\boldsymbol{f}_{\boldsymbol{i}}}{\operatorname{minimize}} & \left\|\boldsymbol{f}_{\boldsymbol{i}}\right\|_{1} \\
\text { subject to } & \left\|\boldsymbol{y}_{\boldsymbol{i}}-\boldsymbol{\Omega}_{\boldsymbol{i}} \cdot \boldsymbol{f}_{\boldsymbol{i}}\right\|_{2}<\delta \text {, and } \delta>0
\end{array}
$$

This program can be written in the following form with a $\ell_{2}$ regularization term:

$$
\begin{array}{ll}
\underset{\boldsymbol{f}_{\boldsymbol{i}}}{\operatorname{minimize}} & \left\|\boldsymbol{f}_{\boldsymbol{i}}\right\|_{1}+\beta\left\|\boldsymbol{y}_{\boldsymbol{i}}-\boldsymbol{\Omega}_{\boldsymbol{i}} \cdot \boldsymbol{f}_{\boldsymbol{i}}\right\|_{2} \\
\text { subject to } & \beta>0
\end{array},
$$

for every point $\boldsymbol{y}_{\boldsymbol{i}}$ in $\left\{\boldsymbol{y}_{\boldsymbol{i}}\right\}_{i=1}^{N} \cdot \beta$ is the parameter that controls the trade-off between sparsity and reconstruction fidelity. To collectively optimize all of the data points, form the matrix $\boldsymbol{Y}=\left[\boldsymbol{y}_{1}, \boldsymbol{y}_{2}, \ldots, \boldsymbol{y}_{N}\right]$ and normalize the recovered coefficients. The optimization problem is now

where $\boldsymbol{Z} \in \mathbb{R}^{N \times N}$ is the matrix of the sparse linear dependence coefficients whose $i$-th column corresponds to the sparse representation of $\boldsymbol{y}_{\boldsymbol{i}}$. Different columnes of $\boldsymbol{Z}$ are independent. Here, the norm $\|\cdot\|_{p}$ of a matrix is the sum of the $\ell_{p}$ vector norms of the columns.

Like the $\ell_{1}$-norm, the $\ell_{2}$-norm also captures the linear dependence relationship since points closer to each other in $\ell_{2}$-norm sense are more likely to be linearly dependent. Combining the two norms into the optimization leads to a group-sparsity optimization:

$$
\begin{array}{ll}
\underset{\boldsymbol{Z}}{\operatorname{minimize}} & \|\boldsymbol{Z}\|_{1}+\alpha\|\boldsymbol{Z}\|_{2}+\beta \| \boldsymbol{Y} \boldsymbol{Z}-\boldsymbol{Y} \\
\text { subject to } & \sum_{i} z_{i j}=1 ; j=1,2,3, \ldots \\
& \operatorname{diag}(\boldsymbol{Z})=\mathbf{0} \\
& \alpha>0, \beta>0
\end{array}
$$

In the ideal case, solving the optimization program (8) recovers the sparse linear dependence coefficients corresponding to the embedded subspaces, which will be used for segmentation in the next step.

\subsection{Subspace segmentation via spectral clustering}

Once the data-driven representation for each data point is found, identification of the common underlying subspaces is the next step. This process of segmenting the linear subspaces from the recovered linear dependence coefficients involves constructing a weighted similarity
Algorithm 1: Point cloud segmentation w.r.t. sparse linear subspaces

Data: PCD $\mathcal{Y}$, arranged as columns of $\boldsymbol{Y} \in \mathbb{R}^{D \times N}$, which is a union of $m$ linear subspaces

Result: Partitions $\boldsymbol{y}_{1}, \boldsymbol{y}_{2}, \ldots, \boldsymbol{y}_{\boldsymbol{m}}$ lying in different subspaces

\section{Begin:}

1. Solve the group-sparsity optimization program for the $N \times N$ matrix $\boldsymbol{Z}$

$$
\begin{array}{ll}
\underset{\boldsymbol{Z}}{\operatorname{minimize}} & \|\boldsymbol{Z}\|_{1}+\alpha\|\boldsymbol{Z}\|_{2}+\beta\|\boldsymbol{Y} \boldsymbol{Z}-\boldsymbol{Y}\|_{2} \\
\text { subject to } & \sum_{i} z_{i j}=1 ; j=1,2,3, \ldots \\
& \operatorname{diag}(\boldsymbol{Z})=\mathbf{0} \\
& \alpha>0, \beta>0
\end{array}
$$

2. Use matrix $Z$ to construct a balanced graph $\boldsymbol{G}=(\boldsymbol{V}, \mathcal{E}, \boldsymbol{W})$. The vertices $\boldsymbol{V}$ are the $N$ data points, and edges $\left(v_{1}, v_{j}\right) \in \boldsymbol{E}$ are with weight $w_{i j} \neq 0$. Compute the adjacency matrix

$$
\boldsymbol{W}_{(\gamma)}=\left|\boldsymbol{Z}_{(\gamma)}\right|+\left|\boldsymbol{Z}_{(\gamma)}^{\top}\right|
$$

with the $\gamma$ largest coefficients.

3. Use $\boldsymbol{W}$ as the adjacency matrix and perform the spectral clustering;

3.1. Construct matrix $\boldsymbol{D}$ with $(i, i)-$ element be the sum of $\boldsymbol{W}$ 's $i$-th row;

3.2. Compute normalized Laplacian

$$
\boldsymbol{L}=\boldsymbol{D}^{-1 / 2} \boldsymbol{W}_{(\gamma)} \boldsymbol{D}^{-1 / 2}
$$

3.3. Perform eigen-decomposition to $L$ and get the first $k$ eigenvectors $\boldsymbol{u}_{1}, \boldsymbol{u}_{2}, \ldots, \boldsymbol{u}_{\boldsymbol{k}}$;

3.4. Form $\boldsymbol{U}=\left[\boldsymbol{u}_{1}\left|\boldsymbol{u}_{2}\right| \ldots \mid \boldsymbol{u}_{\boldsymbol{k}}\right] \in \mathbb{R}^{N \times k}$;

3.5. Form the matrix $\boldsymbol{T} \in \mathbb{R}^{N \times k}$, such that

$$
t_{i j}=\frac{u_{i j}}{\sqrt{\sum_{k} u_{i k}^{2}}} ;
$$

3.6. Let $\left\{\widehat{\boldsymbol{y}}_{i}\right\}_{i=1,2, \ldots, N} \in \mathbb{R}^{k}$ be the vector in the eigen-space corresponding to the $i$-th row of $\boldsymbol{T}$;

3.7. Cluster the points $\widehat{\boldsymbol{y}}_{\boldsymbol{i}}$ with the meanshift algorithm, and retrieve the segmentations $\left\{\boldsymbol{y}_{1}, \boldsymbol{y}_{2}, \ldots, \boldsymbol{y}_{m}\right\}$ in $\mathbb{R}^{3}$ for $\boldsymbol{y}$.

graph $\mathcal{G}=(\boldsymbol{V}, \mathcal{E}, \boldsymbol{W})$ capturing the linear dependence relationships. The $N$ nodes in $\boldsymbol{V}$ of $\mathcal{G}$ correspond to the $N$ input points; the set of edges $\mathcal{E}$ fully connect every two nodes $v_{i}$ and $v_{j}$ with the weight $w_{i j}=\left|z_{i j}\right|+\left|z_{j i}\right|$, where $w_{i j}$ is an element of the adjacency matrix $\boldsymbol{W}$ and $z_{i j}$ is an element of the sparse linear dependence coefficient matrix $\boldsymbol{Z}$. For robustness to noise in the data, when building the similarity graph only the $\gamma$ largest linear dependence 
coefficients should be kept for each point. Accordingly, the adjacency matrix $\boldsymbol{W}_{(\gamma)}$ is expressed as $\boldsymbol{W}_{(\gamma)}=\left|\boldsymbol{Z}_{(\gamma)}\right|+$ $\left|\boldsymbol{Z}_{(\gamma)}^{\top}\right|$, where $\boldsymbol{Z}_{(\gamma)}$ means the matrix with only the $\gamma$ largest coefficients kept for each column with all others set to zero.

Using the adjacency matrix $\boldsymbol{W}_{(\gamma)}$, apply the normalized spectral clustering algorithm (Ng et al., 2002) to cluster the PCD with respect to the linear subspaces. Given the points set $\boldsymbol{Y}=\left[\boldsymbol{y}_{1}, \boldsymbol{y}_{2}, \ldots, \boldsymbol{y}_{N}\right] \in \mathbb{R}^{k}$ with adjacency matrix $\boldsymbol{W}_{(\gamma)}$, define $\boldsymbol{D}$ to be the diagonal matrix whose $(i, i)$-element is the sum of $\boldsymbol{W}_{(\gamma)}$ 's $i$-th row. Construct the Laplacian

$$
\boldsymbol{L}=\boldsymbol{D}^{-1 / 2} \boldsymbol{W}_{(\gamma)} \boldsymbol{D}^{-1 / 2}
$$

Then perform eigen-decomposition on $\boldsymbol{L}$ and use the $k$ (we choose $k=3$ for $\mathrm{PCD}$ in $\mathbb{R}^{3}$ ) largest eigenvectors $\boldsymbol{u}_{1}, \boldsymbol{u}_{2}, \ldots, \boldsymbol{u}_{k}$ of $\boldsymbol{L}$ to form an eigenspace matrix $\boldsymbol{U}=$ $\left[\boldsymbol{u}_{1}\left|\boldsymbol{u}_{2}\right| \ldots \mid \boldsymbol{u}_{\boldsymbol{k}}\right] \in \mathbb{R}^{N \times k}$ by stacking $\boldsymbol{u}_{\boldsymbol{i}}$ in columns. Thirdly a matrix $\boldsymbol{T} \in \mathbb{R}^{N \times k}$ is formed from $\boldsymbol{U}$ by normalizing each row to be of unit norm, such that

$$
t_{i j}=\frac{u_{i j}}{\left(\sum_{k} u_{i k}^{2}\right)^{1 / 2}}
$$

for $i=1,2, \ldots, n$. Let $\boldsymbol{y}_{\boldsymbol{i}} \in \mathbb{R}^{k}$ be the vector corresponding to the $i$-th row of $\boldsymbol{T}$. Lastly, perform meanshift clustering (Comaniciu et al., 2002) on the points $\boldsymbol{y}_{\boldsymbol{i}}$ to get a segmentation result.

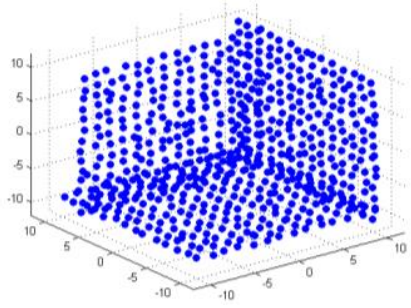

(a) Original PCD

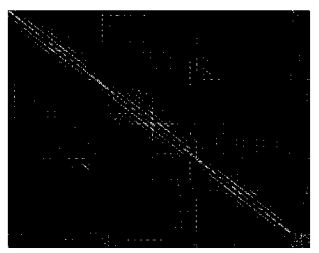

(e) Linear dependence adjacency matrix (with 10 largest coefficients for each point)

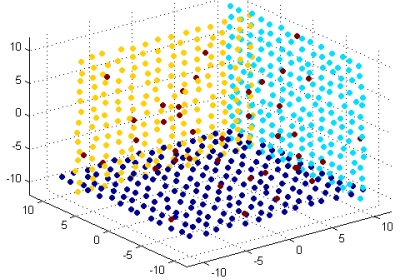

(b) Ground truth for PCD segmentation

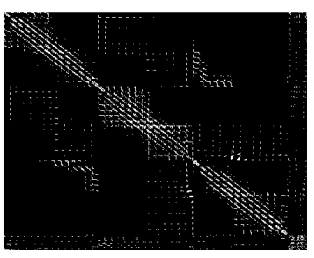

(f) Linear dependence adjacency matrix (with 20 largest coefficients for each point)

\subsection{Illustration of procedure on a synthetic PCD}

The proposed PCD segmentation is summarized in Algorithm 1. To illustrate how the algorithm works, this section details the procedure for a synthetic PCD. The synthetic PCD has 628 points with 588 points from three intersecting planes (196 points per plane) and 40 randomly scattered points. Moreover, all points are corrupted by Gaussian noise with 0.01 variance. The PCD is shown in Figure 3(a), and the ground truth for the PCD segmentation is shown in Figure 3(b) where points from the distinct embedded planes plotted with distinct colors.

The PCD is processed using the proposed algorithm. First, solving the group-sparsity optimization program in Equation (8) with $\alpha=\beta=1$ results in the group-sparse linear dependence coefficients. The matrix containing the $628 \times 628$ linear dependence coefficients is visualized in Figure 3(c), in which the non-zero coefficients (meaning linear dependence) are plotted in white color while the zero coefficients (meaning linear independence) are plotted in black color. Each row in the coefficient matrix stands for one $\mathbb{R}^{3}$ point, and each $1 \times 628$-dimensional row vector consists of the linear dependence coefficients (diagonal elements of the matrix are zero). Compared to the ideal result shown in Figure 3(d), the recovered coefficients matrix is $77.87 \%$ accurate. The accuracy is computed by comparing the two coefficient matrices in Figure 3(c) and (d). The white elements are assigned to 1 and black elements to 0 for both matrices in 3(c) and 3(d), to give the

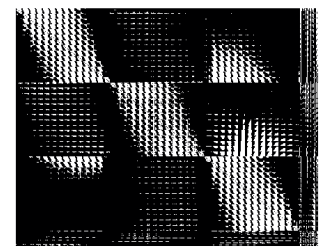

(c) Recovered sparse linear dependence coefficients (the

$\boldsymbol{Z}$ matrix) (black: zero values; white: non-zero values)

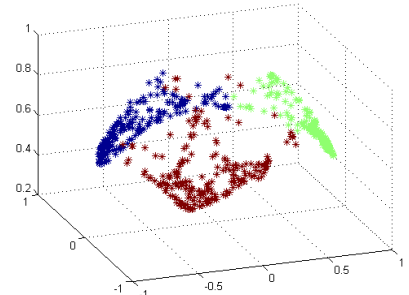

(g) Clustering result in eigenspace in spectral clustering step

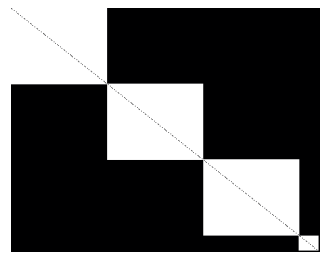

(d) Ideal result of linear dependence coefficients (black: linearly independent; white: linearly dependent)

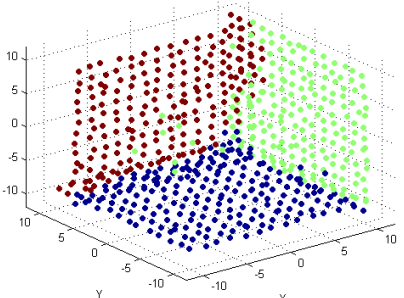

(h) Retrieved segmentation result in $\mathbb{R}^{3}$

Figure 3 Illustration of Algorithm 1 on a synthetic PCD 
matrices $\boldsymbol{M}_{c}$ and $\boldsymbol{M}_{\boldsymbol{d}}$ respectively. Let $\widetilde{\boldsymbol{M}}=\left|\boldsymbol{M}_{\boldsymbol{c}}-\boldsymbol{M}_{\boldsymbol{d}}\right|$, then the accuracy is $\sum_{i, j} \widetilde{\boldsymbol{M}}_{i j} / N^{2}$. Compared to the groupsparsity formulation, the BPDN formulation of Equation (7) has a lower accuracy level of $68.71 \%$.

Rather than use the full matrix, the procedure indicates that the matrix with only the first $\gamma(\gamma<628)$ largest coefficients should be used. In addition to increasing robustness to noise, the decimated matrix $\boldsymbol{W}_{(\gamma)}$ reduces the computational complexity of the spectral clustering step. Figure 3(e) and Figure 3(f) show $\boldsymbol{W}_{(\gamma)}$ with $\gamma=10$ and $\gamma=20$, respectively (visualized by displaying non-zero value elements as white and zero value elements as black). From Figure 3(e) and Figure 3(f), it can be observed that the larger coefficients are nearer to the diagonal elements, while the smaller coefficients are further from the diagonal elements. There is no universal criterion for how many coefficients should be used in constructing the adjacency matrix, but the observations are: if less coefficients are used, then less linear dependence relationships are captured but the algorithm is more robust to noise and has lower computational complexity. For the following steps of the experiment, $\gamma$ is set to be 10 , because this is small enough to generate a sparse adjacency matrix but large enough to capture the linear dependence bases. Further discussion is included in Section 7.3.

By following the remaining steps described in Algorithm 1 , an eigenspace point-set can be obtained, which lies in a simplex structure, as shown in Figure $3(\mathrm{~g})$. Cluster the eigenspace point-set using mean-shift. The clustering result is shown in Figure 3(g) with different clusters plotted in different colors. The PCD segmentation step is finished by assigning the cluster memberships of each point in the eigenspace to the original $\mathbb{R}^{3}$ points. The final segmentation result is plotted in Figure 3(h). The segmentation step achieves $89.46 \%$ accuracy for the points from the underlying planes. Most of the misclassifications occur around the intersecting areas of the planes. The classification accuracy will be further improved in the subsequent steps.

\section{PLANE DETECTION AND MODEL ESTIMATION VIA MAXIMUM LIKELIHOOD SAMPLE CONSENSUS}

The previous step gives a segmentation of the PCD but not the plane model, with some data points potentially misclassified. After the segmentation step, ideally within each segmented group there is at most one linear subspace, meaning that there is one or zero planes in each group. A robust detection and estimation step is needed to determine whether each segmented group arises from a planar subspace, and if so, to estimate the parametric planar model. Moreover, after model estimation, all of the plane models are used to correct potential false segmentations.

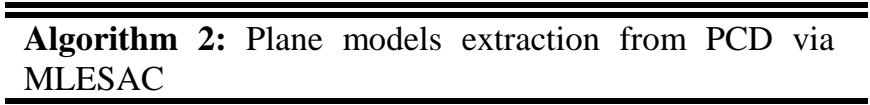

Data: One segmented group $\boldsymbol{y}$ of PCD

Result: Estimated plane parametric models with the inlier set, or failure to find a fit for the model

\section{Begin:}

Repeat

1. Randomly sample 3 points $\left\{\boldsymbol{p}_{\boldsymbol{i}}\right\}_{i=1,2,3}$ with the corresponding homogeneous coordinates $\left\{\boldsymbol{q}_{i}\right\}$. Form matrix

$$
M=\left[q_{1}, q_{2}, q_{3}\right]
$$

2. Perform Singular Value Decomposition $\boldsymbol{U} \boldsymbol{S} \boldsymbol{V}^{\top}=\boldsymbol{M}$, obtain the parameters vector for the plane model as from the last column of $\boldsymbol{V}$

3. Determine the inlier and outlier sets, and the corresponding errors

4. Compute the loss of the model

$$
\begin{aligned}
& P(e \mid \text { Model })=\Upsilon \frac{1}{\sqrt{2 \pi \sigma^{2}}} \exp \left(-\frac{e^{2}}{2 \sigma^{2}}\right)+(1-\Upsilon) \frac{1}{v} . \\
& \text { If } P(e \mid \text { Model })>\text { Threshold } \\
& \text { Then Re-fit the model with inlier set. }
\end{aligned}
$$

Until iterations $>\frac{\log \alpha}{\log \left(1-\gamma^{\Gamma} \text { min }\right)}$

If No verified model extracted

| Output failure to find a fit for the model

else

Output the estimated parametric model with the smallest $L_{M L E S A C}(e)$.

Because the data is noisy and the segmentation result from the previous step may not be accurate, the detection and estimation algorithm in this step should be robust to both noise and false segmentation, which is traditionally done with RANSAC. Traditional RANSAC verifies the estimated models by thresholding the number of inliers. However, in the case of extracting models from PCDs, the cardinality of each segmented point-set varies, meaning that a predefined threshold is not suitable for each group. Compared to RANSAC, the Maximum Likelihood Sample Consensus (MLESAC) algorithm (Torr et al., 2000) adopts the same sampling strategy as RANSAC but chooses the solution by minimizing the probabilistic loss rather than the number of inliers. Minimizing probabilistic loss renders the model verification threshold value invariant to the cardinality of the model's data set. MLESAC is reported to be of higher accuracy and robustness than RANSAC (Choi et al., 2009). Therefore, MLESAC is more suitable for model extraction from PCDs. 
Table 1

Experiment results, ground truth and absolute errors of the model estimation step in the synthetic PCD experiment (the plane models are evaluated using the normalized plane equation $\alpha x+\beta y+\gamma z-1=0$.)

\begin{tabular}{c|c|ccc}
\hline \multirow{2}{*}{ Planes } & \multirow{2}{*}{ Item } & \multicolumn{3}{c}{ Parameters of Plane Models } \\
\cline { 2 - 4 } & & $\alpha$ & $\beta$ & $\gamma$ \\
\hline \multirow{3}{*}{ Plane 1 } & Experiment Result & 0.0001 & 0.1424 & 0.0004 \\
& Ground Truth & 0 & $1 / 7$ & 0 \\
& Absolute Error & 0.0001 & 0.0005 & 0.0004 \\
\hline \multirow{3}{*}{ Plane 2 } & Experiment Result & 0.1001 & 0.0001 & 0.0004 \\
& Ground Truth & 0.1 & 0 & 0 \\
& Absolute Error & 0.0001 & 0.0001 & 0.0004 \\
\hline \multirow{2}{*}{ Plane 3 } & Experiment Result & -0.0001 & -0.0001 & -0.1112 \\
& Ground Truth & 0 & 0 & $-1 / 9$ \\
& Absolute Error & 0.0001 & 0.0001 & 0.0001 \\
\hline
\end{tabular}

\subsection{Planes detection and estimation from PCDs}

MLESAC first randomly samples a subset of points with the minimum cardinality $\Gamma_{\min }$ needed for model estimation, then the sampled subset is used to fit a parametric model. For plane estimation, $\Gamma_{\min }=3$. Denote the three points sampled by $\left\{\boldsymbol{p}_{i}\right\}_{i=1,2,3} \in \mathbb{R}^{3 \times 1}$, then the plane parameters are obtained from the following steps. First express $\left\{\boldsymbol{p}_{\boldsymbol{i}}\right\}$ in homogeneous form as $\boldsymbol{q}_{\boldsymbol{i}}=\left[\boldsymbol{p}_{\boldsymbol{i}}^{\top}, 1\right]^{\top}$, then form the matrix

$$
\boldsymbol{M}=\left[\boldsymbol{q}_{1}, \boldsymbol{q}_{2}, \boldsymbol{q}_{3}\right] \in \mathbb{R}^{4 \times 3} .
$$

Perform singular value decomposition of $\boldsymbol{M}$, which estimates both the normal and the offset of the plane:

$$
\boldsymbol{U} \boldsymbol{S} \boldsymbol{V}^{\top}=\boldsymbol{M} \text {. }
$$

The hypothesized parameter vector $[\alpha, \beta, \gamma,-1]^{\top}$ of the plane is obtained from the fourth column of $V$ with normalization by dividing the additive inverse of the last element.

MLESAC evaluates the fitness of the hypothesis using a probabilistic model for the errors arising from inliers and outliers. The inlier error is modeled as unbiased Gaussian distribution while the outlier error as uniform distribution. Hence the probability of the error given the estimated model is:

$$
P(e \mid \text { Model })=\Upsilon \frac{1}{\sqrt{2 \pi \sigma^{2}}} \exp \left(-\frac{e^{2}}{2 \sigma^{2}}\right)+(1-\Upsilon) \frac{1}{v}
$$

where $e$ is the inlier error, $\Upsilon$ is the prior probability of being an inlier (the ratio of inlier), $v$ is the size of available error space, $\sigma$ is the standard deviation of Gaussian noise. If $P(e \mid M o d e l)$ is larger than the threshold, then the model will be re-estimated using only the inliers and MLESAC terminates. Otherwise, repeat the process with another random sample set, compute the loss, and determine if a further iteration is needed. The maximum number of iterations to perform is

$$
T=\frac{\log \alpha}{\log \left(1-\gamma^{\Gamma_{\min }}\right)}
$$

where $\alpha$ is the estimated failure probability of picking up inlier samples at least once. The MLESAC loop terminates when the required iterations have been finished.

The plane model estimation step is summarized in Algorithm 2. The estimation results of this step correct erroneously segmented points from the previous step by relabeling each point to the model with the minimum Euclidean distance between the point and the model. Pairs of estimated models are merged togethor if the parametric models are close and the point set supports are adjacent.

\subsection{Illustration of Algorithm 2 on the synthetic PCD}

Algorithm 2 is illustrated using the same synthetic PCD discussed in Section 4.3. In the plane models extraction step, points from each group are processed using Algorithm 2. For MLESAC, the threshold for the probability $P(e \mid$ Model $)$ is set to be 0.5 , which is optimized empirically. The inlier set and outlier set detected for each segmented group are plotted in Figure 4(a), in which black ' + ' signs stand for inliers and red ' + ' signs stand for outliers. The models extracted for the three groups are reported in Table 1 with the absolute errors computed by comparing to the ground truth. It can be concluded that the planar models extracted are of high accuracy. These extracted models are further used as feedback to improve the segmentation results by assigning all the points to the model to which the perpendicular Euclidean distance is the smallest among all the models and smaller than a predefined threshold (in this experiment the threshold is 0.1 ), and the points whose perpendicular distances are larger than the threshold are labeled as noise. The segmentation result after this assignment is illustrated in Figure 4(b), which has $93.79 \%$ accuracy for the whole PCD. 


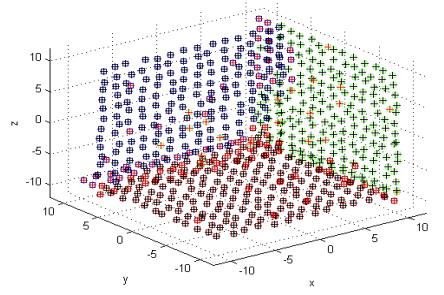

(a) Inlier (black ' + ') and outlis (red ' + ') set obtained in MLESAC

Figure 4: Illustration of Algorithm 2 on the synthetic PCD

\section{Determine Plane Boundaries via QR Decomposition based Projected $\alpha$-Shape}

After the previous two steps, the segmentation of the points from different planes and the corresponding planar models have been obtained. Generating the final representations for the planar patches requires identifying the boundary each extracted planar patch. The challenges of this step are: (1) the boundary point-sets may not be convex; instead they may be concave or even with openings inside the outer "boundary"; (2) points in each point-set may not be uniformly distributed; and (3) the points are corrupted with noise.

\subsection{Maximum projected variance $\alpha$-shape algorithm}

To resolve these problems, some methods have been proposed for determining the boundary point-set. (TruongHong et al., 2013) proposed to combine an angle criterion and the Flying Voxel method for boundary determination, but it works better in less dense point-sets $(<175$ points $/ \mathrm{m}^{2}$ ). The $\alpha$-shape algorithm (Akkiraju et al., 1995) is effective at determining the boundary of large point-sets. The algorithm has been successfully applied to boundary extraction of roof planes from aerial view generated PCDs (Dorninger et al., 2008). Because roofs are of small angle w.r.t. the ground, $3 \mathrm{D}$ points are simply projected onto the grouped plane and then $\alpha$-shape is applied to extract boundary. In this method, no selection of projecting plane are needed and the projecting plane is simply set as the ground plane. In our case the poses (positions and orientations) of planes are arbitrary. Prior to applying the 2D $\alpha$-shape algorithm to 3D planes in arbitrary poses, the $3 \mathrm{D}$ points of planes should be transformed to a $2 \mathrm{D}$ coordinate representation. While there are a variety of ways to construct an orthogonal frame for the plane, we describe here a QR decomposition approach based on the estimated plane normal.

Given a PCD point-set $\boldsymbol{y} \in \mathbb{R}^{n \times 3}$ and its estimated normal $\boldsymbol{n} \in \mathbb{R}^{3 \times 1}$, first a $\mathbb{R}^{3 \times 3}$ matrix $\boldsymbol{A}=\left[\boldsymbol{n}\left|\boldsymbol{v}_{\mathbf{1}}\right| \boldsymbol{v}_{\mathbf{2}}\right]$ is formed, where $\boldsymbol{v}_{\mathbf{1}}, \boldsymbol{v}_{\mathbf{2}} \in \mathbb{R}^{3 \times 1}$ are random column vectors generated from the point-set. Then $\mathrm{QR}$ decomposition of $\boldsymbol{A}$ is:

$$
\boldsymbol{A}=\boldsymbol{Q R}
$$

where $\boldsymbol{Q}=\left[\boldsymbol{Q}_{\mathbf{1}}\left|\boldsymbol{Q}_{\mathbf{2}}\right| \boldsymbol{Q}_{\mathbf{3}}\right] \in \mathbb{R}^{3 \times 3}$ is an orthogonal matrix. The natural coordinate vectors are given by the three column vectors of $\boldsymbol{Q}_{1}, \boldsymbol{Q}_{2}, \boldsymbol{Q}_{3} \in \mathbb{R}^{3 \times 1}$. In this work, the $Z$ - axis in the natural coordinate frame is defined with $\boldsymbol{Q}_{\mathbf{1}}$ (the plane normal), $X-$ axis with $\boldsymbol{Q}_{2}$ and $Y-$ axis with $\boldsymbol{Q}_{3}$. Then project $\boldsymbol{Y}$ onto the natural coordinates by

$$
\widehat{\boldsymbol{y}}^{\prime}=\left[\begin{array}{ll}
\mathbf{I}_{2 \times 2} & \mathbf{0}_{2 \times 1}
\end{array}\right] \cdot\left[\boldsymbol{Q}_{2}\left|\boldsymbol{Q}_{3}\right| \boldsymbol{Q}_{1}\right]^{-1} \cdot \boldsymbol{y}^{\top}
$$

where the factor $\left[\begin{array}{ll}\mathbf{I}_{\mathbf{2} \times \mathbf{2}} & \mathbf{0}_{\mathbf{2} \times \mathbf{1}}\end{array}\right]$ projects $3 \mathrm{D}$ points to $2 \mathrm{D}$ points. The arrangement of columns in $\left[\boldsymbol{Q}_{\mathbf{2}}\left|\boldsymbol{Q}_{\mathbf{3}}\right| \boldsymbol{Q}_{\mathbf{1}}\right]$ performs a $\mathcal{S O}(3)$ transformation aligning the normal vector of plane in the original frame to the $Z$ - axis in the projected frame.

The projected point-set $\widehat{\boldsymbol{y}} \in \mathbb{R}^{n \times 2}$ is obtained by $\widehat{\boldsymbol{y}}=\widehat{\boldsymbol{y}}^{\prime \top}$. The overall algorithm for plane projection and boundary detection via $\alpha$-shape is found in Algorithm 3. The $\alpha$-shape algorithm is then performed on $\widehat{\boldsymbol{y}}$. Since the boundary detected depends on the radius of the circles (or $\alpha$ value),

\begin{tabular}{l}
\hline \hline Algorithm 3: Plane boundary detection via maximum \\
projected variance $\alpha$-shape algorithm
\end{tabular}

Data: A PCD point-set $\boldsymbol{y} \in \mathbb{R}^{n \times 3}$ on a detected plane and the estimated plane normal $\boldsymbol{n} \in \mathbb{R}^{3 \times 1}$

Result: 3D boundary point-set $\boldsymbol{P}_{\boldsymbol{S}}$

Begin:

1. Form a matrix

$$
\boldsymbol{A}=\left[\boldsymbol{n}\left|\boldsymbol{v}_{1}\right| \boldsymbol{v}_{2}\right] \in \mathbb{R}^{3 \times 3} ;
$$

where $\boldsymbol{v}_{\mathbf{1}}, \boldsymbol{v}_{\mathbf{2}} \in \mathbb{R}^{3 \times 1}$ are random column vectors

2. Perform $\mathrm{QR}$ decomposition on $\boldsymbol{A}: \boldsymbol{A}=\boldsymbol{Q R}=$ $\left[Q_{1}\left|Q_{2}\right| Q_{3}\right] \cdot R$

3. Define the natural coordinate frame with $\boldsymbol{Q}_{2}, \boldsymbol{Q}_{3}, \boldsymbol{Q}_{1}$ and project $\mathcal{Y}$ onto the frame by:

$$
\begin{gathered}
\widehat{\boldsymbol{y}}^{\prime}=\left[\mathbf{I}_{\mathbf{2} \times 2} \mid \mathbf{0}_{2 \times 1}\right] \cdot\left[\boldsymbol{Q}_{2}\left|\boldsymbol{Q}_{3}\right| \boldsymbol{Q}_{\mathbf{1}}\right]^{-1} \cdot \boldsymbol{y}^{\top} ; \\
\widehat{\boldsymbol{y}}=\widehat{\boldsymbol{y}}^{\prime \top}
\end{gathered}
$$

where $\widehat{\boldsymbol{y}} \in \mathbb{R}^{n \times 2}$ is the projected point-set

4. Get $\alpha$-shape boundary of $\widehat{\boldsymbol{y}}$;

5. Determining the 3D plane boundary point-set $\boldsymbol{P}_{\boldsymbol{S}}$ by retrieving the membership of the $2 \mathrm{D}$ boundary point-set.

here we set the $\alpha$ value as 3 times of the average single-link 
point-point distance, which is a conclusion assessed experimentally. The boundary detected using this $\alpha$ value is shown in Figure 5(a). The 2D boundaries are then projected back to the 3D space, shown in Figure 5(b). This outside concave boundary is detected without any additional boundaries for the openings inside the point set. performs a $\mathcal{S O}$ (3) transformation aligning the normal vector of plane in the original frame to the $Z$ - axis in the projected frame.

The projected point-set $\widehat{\boldsymbol{y}} \in \mathbb{R}^{n \times 2}$ is obtained by $\widehat{\boldsymbol{y}}=\widehat{\boldsymbol{y}}^{\prime \top}$. The overall algorithm for plane projection and boundary detection via $\alpha$-shape is found in Algorithm 3. The $\alpha$-shape algorithm is then performed on $\widehat{\boldsymbol{y}}$. Since the boundary detected depends on the radius of the circles (or $\alpha$

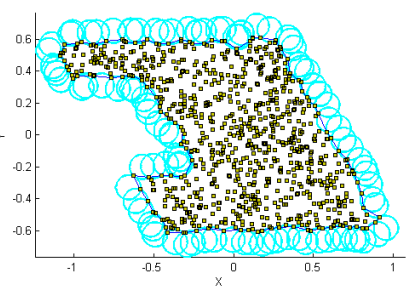

(a) Boundary extracted on the projected 2D point set

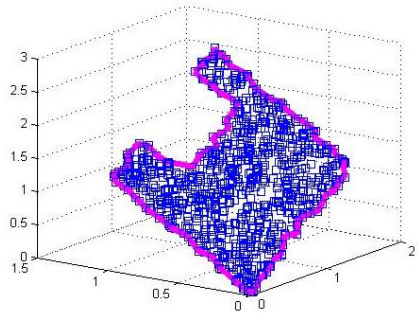

(b) Boundary back-projected to 3D space
Figure 5 Boundaries found using QR decomposition based projected $\alpha$-Shape algorithm (Radius $=3 \times D_{n n}$ )

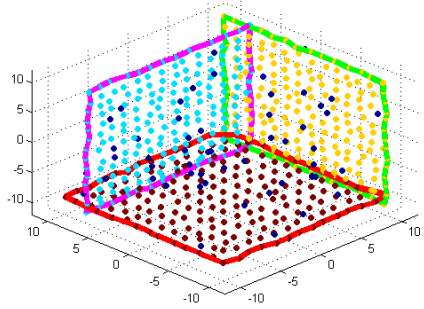

(a) Detected boundaries of extracted planes

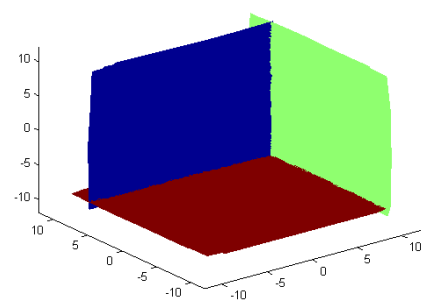

(b) Final planar patches representation
Figure 6: Illustration of Algorithm 3 on synthetic PCD

value), here we set the $\alpha$ value as 3 times of the average single-link point-point distance, which is a conclusion assessed experimentally. The boundary detected using this $\alpha$ value is shown in Figure 5(a). The 2D boundaries are then projected back to the 3D space, shown in Figure 5(b). This outside concave boundary is detected without any additional boundaries for the openings inside the point set.

\subsection{Illustration of Algorithm 3 on a synthetic PCD}

As the final step, the boundaries of each extracted planes are detected by performing Algorithm 3 on each segmented group. The detected boundaries are plotted out in Figure 6(a). Finally, the planar patches representation is generated as shown in Figure 6(b).

\section{EVALUATION OF THE PROPOSED ALGORITHM}

\subsection{Memory and time complexities}

The complexity of the proposed method is analyzed in terms of the memory complexity and computation complexity. The most memory consuming part of the algorithm is the storage of the adjacency matrix $W$ in Algorithm 1. The size of $W$ is at most $2 \gamma N$, where $N$ is the number of points in PCD and $\gamma$ is the number linear dependence coefficients kept. Thus, the memory complexity is $\mathcal{O}(\gamma N)$ with a data structure for sparse matrix/graph, e.g. CSR (Compressed Spared Row Graph). The time complexity is determined by the most expensive steps, which are the group-sparsity optimization programming and the eigen-decomposition steps in Algorithm 1. These steps both require cubic time of the input data size. Since a partitioning strategy is used on the PCD before performing the algorithm (see Section 8.1.2), the time complexity is reduced. Given $k$ as the number of parts the PCD is partitioned into and assume that the PCD is quite uniformly distributed in different parts (and for simplicity, assume each part has roughly the same number of points), the expected running time for each part is $\mathcal{O}\left(\frac{N^{3}}{k^{3}}\right)$. Assuming the $k$ parts are processed sequentially, the final time complexity is $\mathcal{O}\left(\frac{N^{3}}{k^{2}}\right)$. If the $k$ parts are processed concurrently on a parallel machine with $P$ processes, the time complexity is further reduced to $\mathcal{O}\left(\left\lceil\frac{k}{P}\right\rceil \frac{N^{3}}{k^{3}}\right)$ for $P<k$ or $\mathcal{O}\left(\frac{N^{3}}{k^{3}}\right)$ for $P \geq k$.

\subsection{Evaluation metrics}

To evaluate the complete plane identification and extraction algorithm, five different evaluation metrics will be computed. These metrics evaluate different aspects of the algorithm performance to give a comprehensive understanding of how well models detected and extracted. These metrics and their purpose are as follows: Root Mean Square error measures the model fitting accuracy; Normal Deviation measures the orientation accuracy; Unit Volume error measures both the orientation and the translation accuracy; Detection Percentage measures what percentage of the total number of patches were detected; Oversegmentation Factor gives the factor by which the planar models overrepresent the ground-truth models.

\subsubsection{Root mean square error (RMSE)}

The RMSE measures the consistency of the model to the data. For every point $\boldsymbol{X}_{\boldsymbol{i}} \in \mathbb{R}^{3 \times 1}$ that belongs to an extracted plane with the model $\widehat{\boldsymbol{n}}^{\top} \cdot \boldsymbol{X}-d=0$, where $\boldsymbol{n} \in \mathbb{R}^{3 \times 1}$ is the normal of the plane with unit length and $d$ is the offset of the plane. The point-plane distance is then measured by

$$
\text { distance }=\left|\widehat{\boldsymbol{n}}^{\top} \cdot \boldsymbol{X}_{i}-d\right|
$$




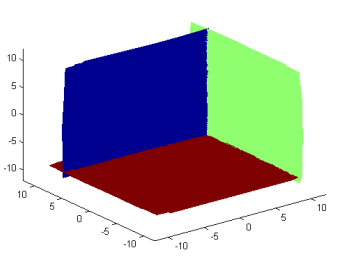

(a) Our method

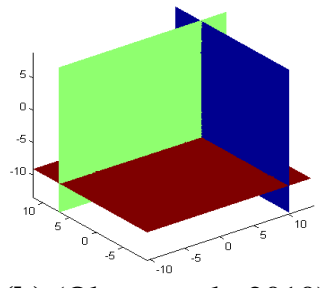

(b) (Okorn et al., 2010)

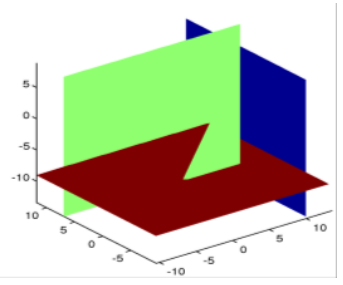

(c) (Budroni et al., 2009)

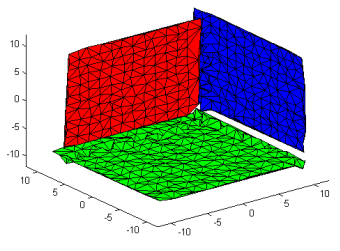

(d) (Adan et al., 2011)

Figure 8 Final results using different methods on the synthetic PCD

Table 2

Evaluation results on the synthetic PCD

\begin{tabular}{l|cccc}
\hline Methods & our method & $\begin{array}{c}\text { (Okorn et al., } \\
\text { 2010) }\end{array}$ & $\begin{array}{c}\text { (Budroni et al., } \\
\text { 2009) }\end{array}$ & (Adan et al., \\
& & $6.91 \pm 1.57$ & $7.95 \pm 2.12$ & $\mathbf{1 . 2 0} \pm \mathbf{1 . 4 6}$ \\
RMSE $(\mathbf{c m})$ & $2.17 \pm 0.61$ & $0.32 \pm 0.08$ & $0.37 \pm 0.11$ & $0.13 \pm 0.25$ \\
Unit Volume Err. $\left(\mathbf{m}^{\mathbf{3}} / \mathbf{m}^{\mathbf{2}}\right)$ & $\mathbf{0 . 1 3} \pm \mathbf{0 . 0 2}$ & $\mathbf{0}$ & $\mathbf{0}$ & $17.98 \pm 21.47$ \\
Normal Deviations $($ degree $)$ & $0.14 \pm 0.12$ & $100 \%$ & $100 \%$ & $100 \%$ \\
Detection Percentage & $\mathbf{1 0 0} \%$ & 1 & 1 & 1 \\
Oversegmentation Factor & 1 & & 1 \\
\hline
\end{tabular}

The root mean square error (RMSE) for each extracted plane is defined as

$$
R M S E=\sqrt{\frac{1}{I} \sum_{i}\left(\widehat{\boldsymbol{n}}^{\top} \cdot \boldsymbol{X}_{i}-d\right)^{2}}
$$

where $i=1,2, \ldots, I$ is the index of the points that associated to the plane.

\subsubsection{Normal deviations}

The normal deviation measures the accuracy of orientation between the extracted plane compared to the ground-truth plane. Given the normal vector $\hat{n}$ of an estimated plane and the corresponding ground-truth normal vector $n$, the normal deviation is:

$$
\text { Normal Deviation }=\operatorname{acos}\left(\widehat{\boldsymbol{n}}^{\top} \cdot \boldsymbol{n}\right)
$$

\subsubsection{Unit volume error}

Besides orientation accuracy, the localization accuracy of the plane is important. Accordingly, here we define an evaluation metric which captures both the orientation and translation accuracy, the unit volume error. It is the volume generated from the estimated patch and the ground-truth patch divided by the area of the ground-truth patch. The volume error is illustrated in Figure 7. The volume is defined in the direction orthogonal to the ground-truth patch. In the calculation of the volume, absolute distances are used instead of the signed distances. The units of this score are $m^{3} / m^{2}=m$.

$$
\text { UnitVolumeError }=\frac{\text { total volume error }}{\text { area of ground truth patch }}
$$

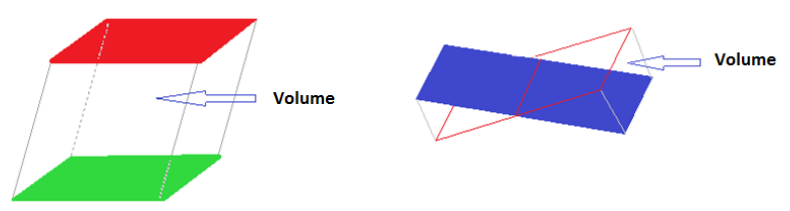

Figure 7 Volume between two planar patches

\subsubsection{Detection percentage}

This metric evaluates how completely the algorithm is able to detect all existing planar patches in the PCD. It is the percentage of the number of extracted patches, relative to the quantity of patches in the ground truth model. The number of extracted patches is defined as the number of patches in the ground-truth data that are correctly found by the algorithm. For example, if there are 20 planar patches in the whole ground-truth PCD, and the algorithm is able to extract 12 out of the 20 planar patches, then the Detection Percentage is $60 \%$. Moreover, if patch $\mathrm{A}$ in the groundtruth data is found but broken into two patches by the algorithm, patch $\mathrm{A}$ is counted as one patch extracted; or if only a part of patch $\mathrm{A}$ is found by the algorithm, it is still counted as one extracted patch. The ideal value is $100 \%$.

\subsubsection{Oversegmentation factor}

This metric aims to evaluate for a detected ground-truth planar patch, how well the procedure models the patch. For each ground-truth planar patch, the number of the corresponding extracted planes is counted. Then the oversegmentation factor is defined to be the quantity of extracted plane models divided by the quantity of unique ground-truth models associated to them. For example, suppose that the procedure detected six planar patches, two 
belonging to one ground truth model, and four belonging to a second ground truth model. Then the oversegmentation factor is $(2+4) / 2=3$. Combining Detection Factor, the ideal case is that the oversegmentation factor equals to 1 and the detection percentage equals to $100 \%$. In this case, there is a one-to-one mapping from the estimated patches to the ground-truth patches.

\subsection{Overall performance of the proposed algorithm on the synthetic PCD}

Using the evaluation metrics, the proposed algorithm is compared to three baseline algorithms. The three baseline methods are the Hough transform based algorithm of (Okorn et al., 2010), the plane-sweeping algorithm of (Budroni et al., 2009), and the region-growing based method of (Adan et al., 2011). Some of these algorithms only address parts of the pipeline of this problem. In order for a fair comparison, the parts in the pipeline which are not solved by the compared algorithms will be addressed using the corresponding steps of the proposed algorithm. Moreover, the final planar patch representations of these methods are different. For method (Okorn et al., 2010) and method (Budroni et al., 2009) the final results are in solid planar patches, while for method (Adan et al., 2011) the final results are segmentations of points. The results of these three methods on the synthetic PCD are shown in Figure 8 respectively. The evaluation results are shown in Table 2, which are presented in the format of "mean \pm standard deviation", because there are multiple planes in the dataset and the statistics are computed over the planes. This simple, synthetic PCD example does not fully reflect realworld PCDs. For example, the real-world dataset may not be oriented precisely, which would introduce errors when using methods in (Okorn et al., 2010) and (Budroni et al., 2009).

In Table 2, the methods (Okorn et al., 2010) and (Budroni et al., 2009) have RMSE $>0$ but the normal deviations are zeros because the extracted planes are of an offset compared to the ground-truth planes but they are also parallel to the ground-truth planes (and this is why the normal deviations are exactly zeros). Note that the normal deviations of (Okorn et al., 2010) and (Budroni et al., 2009)

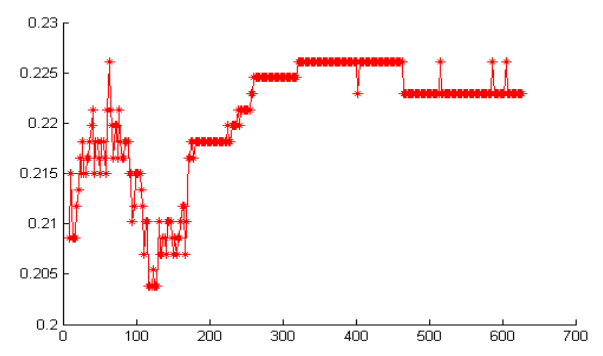

Figure 9 Misclassification rate w.r.t. different numbers of linear elements in constructing the similarity graph can be zeros because in this synthetic example all the planes are placed perfectly parallel to the coordinate planes. These two methods rely on the projection onto coordinate planes or plane-sweeping along the direction from rotational sweeping. Thus, they have zero normal deviations in this synthetic example. However, in reality, the planes in pointclouds may not perfectly align with the coordinate planes or the extracted direction. Therefore in the real-world PCDs example, these two methods do not have zero normal deviations. It is worthy to note that none of these compared methods is able to give estimated plane models or the detailed boundaries. Especially, the region-growing based methods are only able to give segmentation of point clouds that ideally belong to some planes.

We end the discussion for the synthetic PCD experiment by investigating the influence of the number (denoted as $\gamma$ ) of linear dependence coefficients used in constructing the similarity graph. The misclassification rates of the PCD segmentation step w.r.t. $\gamma$ from 2 to 627 are plotted in Figure 9. As it can be observed, the mis-classification rate varies between $20.38 \%$ and $22.61 \%$. Given that the model fitting step corrects this error, the change in performance as a function of $\gamma$ is not significant enough to warrant using large values of the parameter $\gamma$. Thus, it is recommended to use a relatively small $\gamma$, one which would correspond to selecting a small percentage of the total dataset.

\section{EXPERIMENTS AND EVALUATIONS ON REAL- WORLD CIVIL INFRASTRUCTURES PCD}

This section evaluates the performance of the proposed algorithm when applied to two real-world infrastructures PCDs. The real-world PCDs used were captured using two different kinds of methods: a building PCD reconstructed from videos using Structure-from-Motion methods, and a bridge PCD captured directly using a laser scanner. These two real-world PCDs are both of civil infrastructures but with different levels of noise.

When dealing with large-scale PCDs, some preprocessing and post-processing steps can be added to help reduce the processing time. First, the PCD is cut into multiple smaller PCDs by partitioning the volume into smaller volumes. The proposed procedure is applied to each partition. After extracting all planar patch models, the postprocessing step merge the patches that are adjacent and have low difference in the model parameters. These preprocessing and post-processing steps are used in the experiments in Section 8.

\subsection{Experiment 1: building dataset from video}

\subsubsection{Point cloud dataset}

In this experiment, the PCD of a real building is used. The PCD is reconstructed from video using the open-source 3D reconstruction tool PMVS2 (Furukawa et al., 2010). A 
frame from the video is shown in Figure 10. Due to the physical constraints of the environment, only three faces of the building were captured. Moreover, there are some occlusions in the scene, e.g., trees, decorations, etc. The reconstructed raw PCD is displayed in Figure 11. The point cloud consists of 1,681,634 points, with relatively large measurement uncertainty.

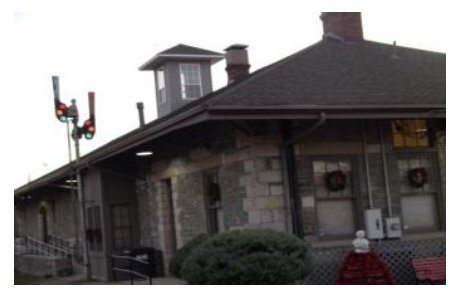

Figure 10 A sample image from video used to reconstruct a building

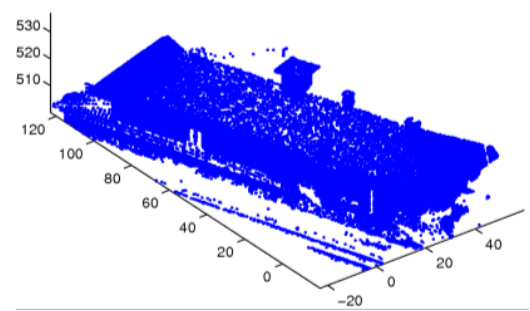

Figure 11 Raw PCD representation of a building

\subsubsection{Experimental results}

The building PCD is processed using the proposed algorithm, with parameter settings as listed in Table 3. To lower the computational complexity, the PCD is first partitioned into $8 \times 8 \times 4=256$ parts. The final result of the experiment, after merging the partition results, is shown

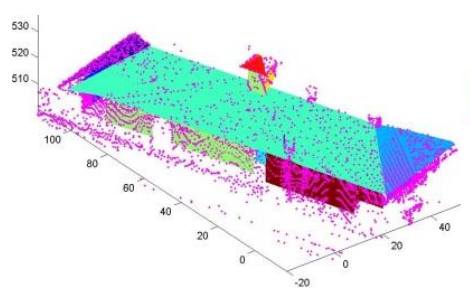

(a) Our method (view 1)

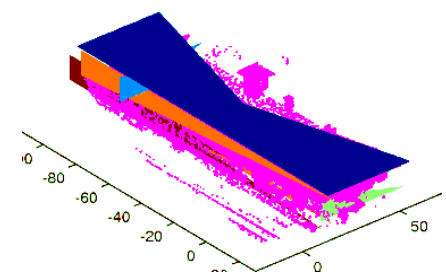

(e) Method in (Budroni et al., 2009) (view 1)

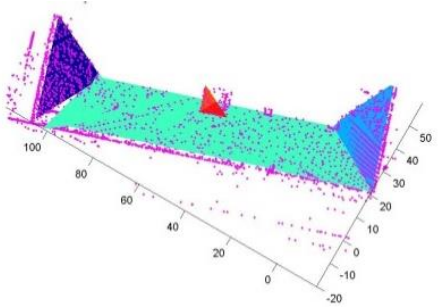

(b) Our method (view 2)

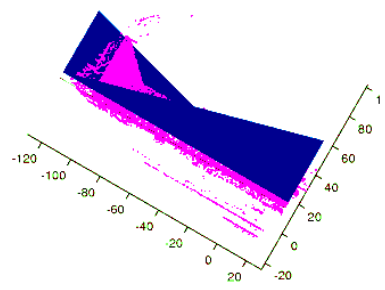

(f) Method in ( Budroni et al., 2009) (view 2)

in Figure 12(a), (b). The algorithm extracts 16 planar patches from the PCD.

The raw PCD is also plotted in Figure 12(a), (b) in magenta to provide intuitive comparison between the raw point cloud and the extracted patches. Note that some open parts (for instance, the intersecting part between two roof planes in Figure 12 (b)) exist because the point cloud itself does not capture the corresponding part due to some occlusions. From the experiment it can be observed that the extracted patches fit with the point cloud very well.

Table 3

Parameter configurations for the building PCD experiment

\begin{tabular}{l|c}
\hline Parameters & Value \\
\hline optimization parameter $\alpha$ & 1 \\
\hline optimization parameter $\beta$ & 1 \\
\hline number of coefficients used in adjacency matrix & 10 \\
\hline MLESAC verification probability threshold & 0.5 \\
\hline $\begin{array}{l}\text { MLESAC false alarm rate } \\
\text { (probability a good minimal sample set never } \\
\text { picked) }\end{array}$ & $1 \mathrm{e}-3$ \\
$\begin{array}{l}\text { MLESAC assumed noise standard deviation } \\
\text { MLESAC minimum iterations }\end{array}$ & 0.1 \\
\hline $\begin{array}{l}\text { Point-model distance threshold for Re- } \\
\text { segmentation }\end{array}$ & 1000 \\
\hline
\end{tabular}

\subsubsection{Comparison to baseline methods}

For (Okorn et al., 2010) method, since proper orientation is required, the orientation of the PCD is corrected to align the walls to coordinate axes before conducting the experiment. The parameters of the compared methods are as follows. For (Okorn et al., 2010), we set the grid size of the 2D histogram as $0.1 \mathrm{~m} \times 0.1 \mathrm{~m}$. For (Budroni et al.,

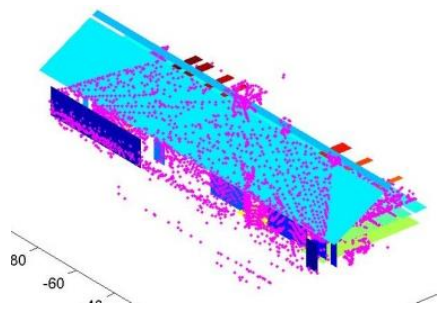

(c) Method in (Okorn et al., 2010) (view 1)

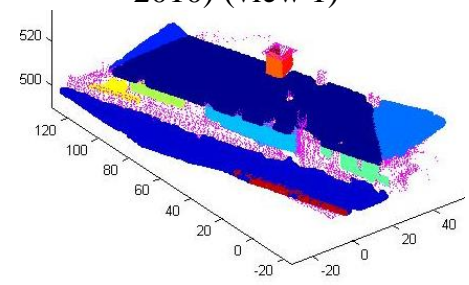

(g) Method in (Adan et al., 2011) (view 1)

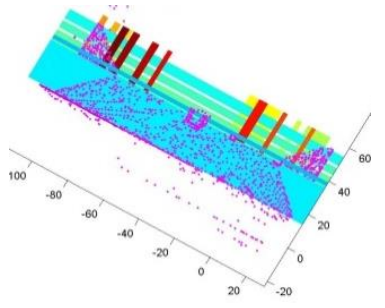

(d) Method in (Okorn et al., 2010) (view 2)

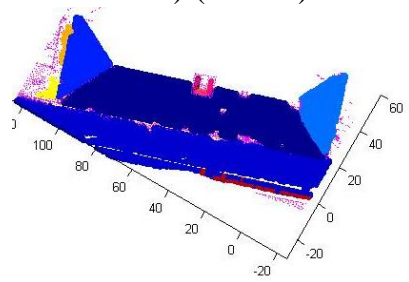

(h) Method in (Adan et al., 2011) (view 2)

Figure 12 Extracted planar patches for the building PCD using different methods, plotted with the raw PCD (in magenta) 
Table 4

Evaluation results on the building PCD

\begin{tabular}{l|cccc}
\hline Methods & our method & $\begin{array}{c}\text { (Okorn } \text { et al., } \\
\text { 2010) }\end{array}$ & $\begin{array}{c}\text { (Budroni } \text { et al., } \\
\text { 2009) }\end{array}$ & (Adan et al., 2011) \\
\hline RMSE $(\mathbf{c m})$ & $2.05 \pm 0.57$ & $7.20 \pm 1.66$ & $\mathbf{0 . 4 7} \pm \mathbf{0 . 2 7}$ & $7.85 \pm 5.33$ \\
Unit Volume Err. $\left(\boldsymbol{m}^{\mathbf{3}} / \mathbf{m}^{\mathbf{2}}\right)$ & $\mathbf{0 . 4 5} \mathbf{0 . 6 3}$ & $10.29 \pm 3.81$ & $4.06 \pm N / A$ & $1.47 \pm 2.42$ \\
Normal Deviations $($ degree $)$ & $\mathbf{0 . 9} \pm \mathbf{0 . 6}$ & $3.52 \pm 0.46$ & $5.04 \pm N / A$ & $3.87 \pm 1.72$ \\
Detection Percentage & $\mathbf{9 3} \%$ & $65 \%$ & $29 \%$ & $71 \%$ \\
Oversegmentation Factor & $\mathbf{1 . 1}$ & 3.5 & 2 & 1.3 \\
\hline
\end{tabular}

2009) method, the number of the bins used to generate the histogram of point numbers for sweeping is 200 and the threshold to define a plane in the histogram is set to be half of the maximum value in the histogram. For (Adan et al., 2011) method, the PCD is voxelized into $2 \mathrm{~cm} \times 2 \mathrm{~cm} \times$ $2 \mathrm{~cm}$ grids. The number of neighbour points for normal estimation is 50; the threshold of maximum angle between normal vectors is 2 degrees; the curvature threshold to guarantee points are well-described by plane models is set as 1 . All of these parameter configurations are optimized empirically.

Ground-truth data of the building is collected using a professional total station (i.e., SOKKIA 30R). Points are measured for each facet of the infrastructures, especially the points that define the boundary of each facet of the infrastructure. The PCD is obtained by merging the pointsets from different scan domains using the software of the total station. Another method of merging point-sets from multiscan domains is proposed in (Sareen et al., 2012) for generating more consistent PCDs. After collecting the PCD, the measured points belonging to each specific facet are selected manually and used to generate the ground-truth data for each facet. The planes measured as ground-truth are shown in Figure 13. These planes are used to evaluate RMSE, unit volume error and normal deviations. For detection percentage and oversegmentation factor, in total 14 planes are considered. The experiment is evaluated using the evaluation metrics defined in Section 7.1.

The evaluation results of the proposed procedure and the three baseline procedures are listed in Table 4 . Note that in Table 4, method (Budroni et al., 2009), no standard deviation is given because the method only extracts one patch that can be considered corresponding to a groundtruth plane, which is the largest wall of the building. Since only one extracted patch is considered to have a corresponding ground-truth patch, we only have one value for each metric and undefined standard deviation.

From Table 4 it can be concluded that the proposed method has the best performance among all the comparative methods.

\subsection{Experiment 2: bridge dataset from laser scanner}

\subsubsection{Point cloud dataset}

This section applies the algorithm to the PCD of a bridge captured using a professional laser scanner (Leica Laser Scan Total Station C10). The bridge span is more than 200 meters. The raw PCD has 2,005,582 points, which are shown in Figure 14. Compared to the building dataset, this PCD is of higher accuracy. Moreover, this PCD has more planar patch elements then the building PCD. In total, there are 40 patches ( 2 for the road surfaces, 2 for the left and right span along the road surface, 9 square columns with 36 planar patches in total). Since the upper surfaces and the lower surfaces coincide with the big upper and lower planes of span of the bridge, we decided to only count the planes of the span instead of the planes of the beams to avoid confusions. The surfaces on the floor and the ramp are not considered in this experiment because they are not parts of the infrastructure components. The proposed procedure and the baseline methods are tested on this PCD.

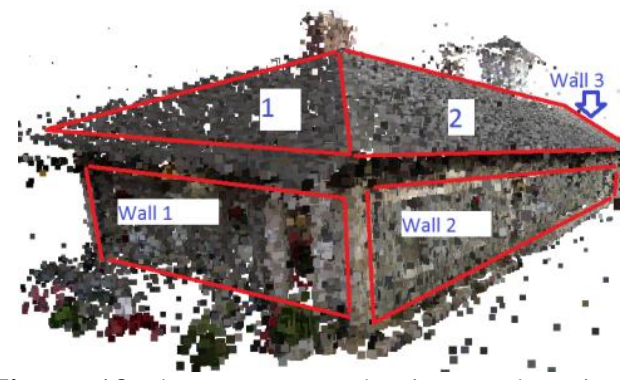

Figure 13 Planes measured using total stations to provide ground truth data

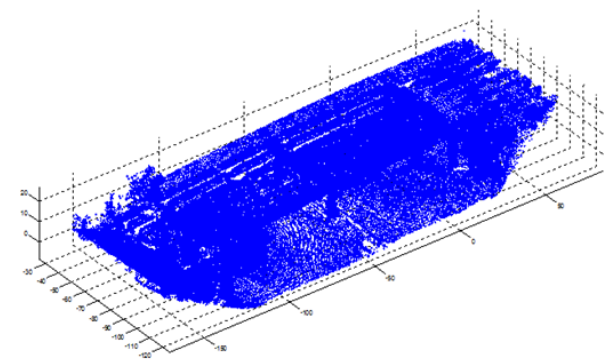

Figure 14 Point-cloud representation of the raw bridge PCD 


\subsubsection{Experimental results}

The results on the bridge PCD using the proposed algorithm and the same partition strategy as in the previous experiment are shown in Figure 15(a), (b). In total there are 29 planar patches extracted excluding the patches for the floor and the ramp. The completeness in terms of the number of planar patches is $31 / 40 \approx 78 \%$. The extracted patches cover the horizontal and the vertical surfaces of the road parts, and most of the surfaces ( 25 out of 36 patches) on all the columns. For the patches of the columns which the algorithm fails to extract, it can be observed from the raw PCD that the point densities for these patches are lower than for the detected column patches, because the positions of these patches are blocked in some of the laser scan views. The ground-truth data of this PCD is generated manually from laser total station data. The performance is validated using the metrics in Section 7.1 and the evaluation statistics are listed in Table 5 in details. It can be concluded from table that the proposed algorithm achieves high accuracy in all of these three metrics, and the result on this PCD is more accurate than the result on the building PCD.

\subsubsection{Comparison to baseline methods}

The parameters configured are as follows. For (OKorn et al, 2010), we set the grid size of the $2 \mathrm{D}$ histogram as $0.3 \mathrm{~m} \mathrm{x}$ $0.3 \mathrm{~m}$. For (Budroni et al, 2009), the parameters are the

same as the previous experiment. For (Adan et al., 2011) method, voxel grid size is $3.5 \mathrm{~cm} \times 3.5 \mathrm{~cm} \times 3.5 \mathrm{~cm}$. The number of neighbour points for normal estimation is 100 ; the angle threshold is 2 degrees; the curvature threshold is 1.5. Again, these parameters were optimized empirically according multiple trails of experiments.

The results of the baseline method are also found in Figure 15. The result of (Okorn et al., 2010) is shown in Figure 15(c) (d), the result of (Budroni et al., 2009) is in Figure 15 (e) (f), and the result of (Adan et al., 2011) is in Figure 15 (g) (h).

Evaluation statistics for all the procedures are listed in Table 5. The proposed method achieves the best performance in terms of all the metrics except for the RMSE. For the RMSE, the method in (Adan et al., 2011) has the smallest mean value, while the RMSE of the proposed method is slightly larger than the method in (Adan et al., 2011) but with smaller standard deviation of RMSE than method in (Adan et al., 2011) which means the RMSEs of all the extracted planes for the proposed method are more consistent than that for method in (Adan et al., 2011). It can also be observed that (Adan et al., 2011) method has larger oversegmentation factor compared to the proposed method, which is because it breaks the bridge surface into several patches. In addition, it is worthy to note

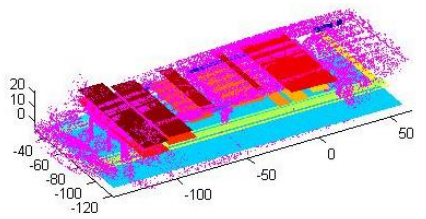

(c) Method in (Okorn et al., 2010) (view 1)

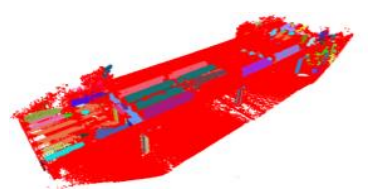

(g) Method in (Adan et al., 2011) (view 1)

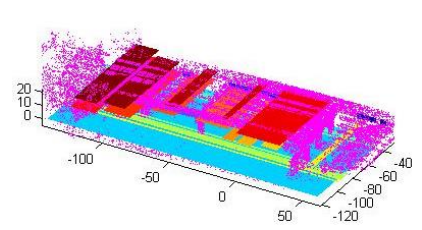

(d) Method in (Okorn et al., 2010) (view 2)

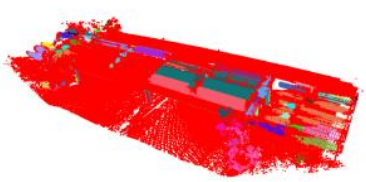

(h) Method in (Adan et al., 2011) (view 2) al., 2009) (view 1)

(f) Method in (Budroni et al., 2009) (view 2)

Figure 15 Extracted planar patches for the bridge PCD using different methods, plotted with the raw PCD (in magenta)

Table 5

Evaluation results on the bridge PCD

\begin{tabular}{l|cccc}
\hline Methods & our method & (Okorn et al., & (Budroni et al., & (Adan et al., \\
& & 2010) & 2009) & 2011) \\
\hline RMSE $(\mathbf{c m})$ & $0.41 \pm 0.11$ & $5.89 \pm 3.90$ & $2.16 \pm 2.44$ & $\mathbf{0 . 3 1} \pm \mathbf{0 . 4 0}$ \\
Unit Volume Err. $\left(\boldsymbol{m}^{\mathbf{3}} / \boldsymbol{m}^{\mathbf{2}}\right)$ & $\mathbf{0 . 0 8} \pm \mathbf{0 . 0 9}$ & $0.66 \pm 0.47$ & $1.65 \pm 0.21$ & $0.92 \pm 0.87$ \\
Normal Deviations $($ degree $)$ & $\mathbf{0 . 1 1} \pm \mathbf{0 . 1 1}$ & $2.50 \pm 0.31$ & $3.71 \pm 1.15$ & $1.17 \pm 2.68$ \\
Detection Percentage & $\mathbf{7 8} \%$ & $10 \%$ & $40 \%$ & $70 \%$ \\
Oversegmentation Factor & $\mathbf{1 . 0 3}$ & 3.6 & 1.5 & 2.1 \\
\hline
\end{tabular}


that (Adan et al., 2011) method gives false positives in the final result and more importantly the outputs of this method are segmentations of the input PCD with no estimates of the plane models nor the planar patch boundaries. In general, it can be concluded the proposed method has better overall performance among all the comparative methods.

\section{CONCLUSION}

This work focuses on the problem of planar model extraction from civil infrastructure PCDs, which requires three objectives including the detection of planar structures, estimation of planar parametric models and determination of the planar model boundaries. In this paper, an innovative algorithm is proposed for addressing this problem. The proposed procedure is demonstrated to be suitable for largescale noisy infrastructure PCDs and able to address all the three objectives. One of the most important steps of this procedure is that it first recovers the linear dependence relationship between each point in the PCD, by solving a group-sparsity inducing optimization program. With the recovered linear dependence coefficients, the algorithm further segments the PCD by clustering the points according to the linear subspace. The clustering uses spectral clustering with a similarity graph formed from the linear dependence coefficients matrix. After PCD segmentation, planes are detected and estimated for each segmented group via an SVD based approach using MLESAC. Finally, the boundary of each plane is detected using the $\alpha$-shape algorithm. The proposed algorithm is tested extensively using three types of PCDs: synthetic data, a PCD of a real building reconstructed from video, and a PCD of a bridge captured directly using laser scanners. For the synthetic PCD experiment, detailed results are provided to illustrate every step of the procedure. To comprehensively evaluate the model extraction performance, five different evaluation metrics are applied. Furthermore, the proposed algorithm is also compared with three baseline methods. The experimental results and the evaluation statistics on the real-world PCDs demonstrate that the proposed algorithm has the best overall performance among the comparative methods on the realworld PCDs. The future work includes the extension of the proposed algorithm for extracting more geometric shapes embedded in PCDs, and recognizing the infrastructure components after model extraction.

\section{REFERENCES}

Adan, A., Xiong, X., Akinci, B., \& Huber, D. (2011), Automatic Creation of Semantically Rich 3D Building Models from Laser Scanner Data. Proceedings of the International Symposium on Automation and Robotics in Construction (ISARC), pp. 342-347.
Agarwal P. \& Mustafa, N. (2004), K-means Projective Clustering, Proceedings of ACM SIGMOD-SIGACTSIGART Symposium Principles of Database Systems, pp. 155-165.

Akkiraju, N., Edelsbrunner, H., Facello, M., Fu, P., Mucke, E. P. \& Varela, C. (1995), Alpha Shapes: Definition and Software, Proceedings of International Computational Geometry Software Workshop, pp. 63-66.

Bosché, F. (2012), Plane-based Registration of Construction Laser Scans with 3D/4D Building Models. Advanced Engineering Informatics, 26(1), pp. 90-102.

Budroni, A. \& Jan B. (2009), Toward Automatic Reconstruction of Interiors from Laser Data, Proceedings of the Workshop on 3D Virtual Reconstruction and Visualization of Complex Architectures.

Choi, S., Kim, T. \& Yu, W. (2009), Performance Evaluation of RANSAC Family, Proceedings of the British Machine Vision Conference.

Comaniciu, D. \& Meer, P. (2002), Mean shift: A Robust Approach Toward Feature Space Analysis, Pattern Analysis and Machine Intelligence, IEEE Transactions on, 24(5), pp. 603-619.

Costeira, J. \& Kanade, T. (1998), A Multibody Factorization Method for Independently Moving Objects, Internaional Journal of Computer Vision, 29(3), pp. 159179.

Davison, A. J., Reid, I. D., Molton, N. D. \& Stasse, O. (2007), MonoSLAM: Real-Time Single Camera SLAM, Pattern Analysis and Machine Intelligence, IEEE Transactions on, 29(6), pp. 1052-1067.

Deshpande, S.S. (2013), "Improved Floodplain Delineation Method Using High Density LiDAR Data," Computer-Aided Civil and Infrastructure Engineering, 28(1), pp. 68-79.

Donoho, D. L. (2006), For Most Large Underdetermined Systems of Linear Equations the Minimal $\ell_{1}$-norm Solution Is Also the Sparsest Solution. Communications on Pure and Applied Mathematics, 59(6), pp. 797-829.

Dorninger, P., \& Pfeifer, N. (2008), A Comprehensive Automated 3D Approach for Building Extraction, Reconstruction, and Regularization from Airborne Laser Scanning Point Clouds. Sensors, 8(11), pp. 7323-7343.

Elhamifar, E. \& Vidal, R. (2009), Sparse Subspace Clustering, Proceedings of the IEEE Conference on Computer Vision and Pattern Recognition, pp. 2790-2797.

Farin, G. (1992), From Conics to NURBS: A Tutorial and Survey, IEEE Computer Graphics and Applications, 12(5), pp. 78-86.

Farin, G. (1996), Curves and Surfaces for Computer Aided Geometric Design: A Practical Guide (4th edition), Academic Press, Boston, Massachusetts.

Fischler, M. A. \& Bolles, R. C. (1981), RANdom SAmple Consensus: A Paradigm for Model Fitting with Applications to Image Analysis and Automated 
Cartography. Communications of the ACM, 24(6), pp. 381395.

Furukawa, Y. \& Ponce, J. (2010), Accurate, Dense, and Robust Multiview Stereopsis, Pattern Analysis and Machine Intelligence, IEEE Transactions on, 32(8), pp. 1362-1376.

Goedert, J., Bonsell, J. \& Samura, F. (2005), Integrating Laser Scanning and Rapid Prototyping to Enhance Construction Modeling, Journal of Architecture Engineering, 11(2), pp. 71-74.

Hähnel, D., Burgard, W. \& Thrun, S. (2003), Learning Compact 3D Models of Indoor and Outdoor Environments with a Mobile Robot, Robotics and Autonomous Systems, 44(1), pp. 15-27.

Huber, D., Akinci, B., Oliver, A., Anil, E., Okorn, B. \& Xiong, X. (2011), Methods for Automatically Modeling and Representing As-built Building Information Models, Proceedings of the NSF CMMI Research Innovation Conference.

Landes, T. \& Grussenmeyer P. (2007), Hough-transform and Extended RANSAC Algorithms for Automatic Detection of 3D Building Roof Planes from LiDAR Data, Science and Technology, 36(1), pp. 407-412.

Liu, G., Lin, Z. \& Yu, Y. (2010), Robust Subspace Segmentation by Low-rank Representation, Proceedings of Internaional Conference of Machine Learning, pp. 663-670

Lu, L. \& Vidal, R. (2006), Combined Central and Subspace Clustering on Computer Vision Applications, Proceedings of International Conference of Machine Learning, pp. 593-600.

Ma, Y., Derksen, H., Hong, W. \& Wright, J. (2007), Segmentation of Multivariate Mixed Data via Lossy Coding and Compression, Pattern Analysis and Machine Intelligence, IEEE Transactions on, 29(9), pp. 1546-1562.

Martín Nevado, M., Gómez García-Bermejo, J., \& Zalama Casanova, E. (2004), Obtaining 3D models of indoor environments with a mobile robot by estimating local surface directions. Robotics and Autonomous Systems, 48(2), pp. 131-143.

Ng, A. Y., Jordan, M. I. \& Weiss, Y. (2002), On Spectral Clustering: Analysis and An Algorithm, Advances in neural information processing systems, pp. 849-856.

Okorn, B., Xiong, X., Akinci, B. \& Huber, D. (2010), Toward Automated Modeling of Floor Plans, Proceedings of the Symposium on 3D Data Processing, Visualization and Transmission.

Pollefeys, M., Nister, D., Frahm, J. M., Akbarzadeh, A., Mordohai, P., Clipp, B., Engels, C., Gallup, D., Kim, S. J., Merrell, P., Salmi, C., Sinha, S., Talton, B., Wang, L., Yang, Q., Stewenius, H., Yang, R., Welch, G. \& Towles, H. (2008), Detailed Real-time Urban 3D Reconstruction from Video, International Journal of Computer Vision, 78(2-3), pp. 143-167.

Rao, S., Tron, R., Ma, Y., \& Vidal, R. (2008), Motion Segmentation via Robust Subspace Separation in the
Presence of Outlying, Incomplete, or Corrupted Trajectories, Proceedings of the IEEE Conference on Computer Vision and Pattern Recognition, pp. 1-8.

Sareen, K. K., Knopf, G. K. \& Canas, R. (2012), Consistent Point Clouds of Narrow Spaces using Multiscan Domain Mapping, Computer-Aided Civil and Infrastructure Engineering, 27(8), pp. 555-638.

Schnabel, R., Wahl, R. \& Klein, R. (2007), Efficient RANSAC for Point-Cloud Shape Detection, Computer Graphics Forum, 26(2), pp. 214-226.

Seitz, S. M., Curless, B., Diebel, J., Scharstein, D. \& Szeliski, R. (2006), A Comparison and Evaluation of MultiView Stereo Reconstruction Algorithms, Proceedings of the IEEE Conference on Computer Vision and Pattern Recognition, pp. 519-528.

Stamos, I. \& Allen, P. E. (2000), 3-D Model Construction using Range and Image Data, Proceedings of the IEEE Conference on Computer Vision and Pattern Recognition, vol. 1, pp. 531-536.

Soltanolkotabi, M. \& Candes, E. J. (2012), A Geometric Analysis of Subspace Clustering with Outliers, The Annals of Statistics, 40(4), pp. 2195-2238.

Tarsha-Kurdi, F., Landes, T. \& Grussenmeyer, P. (2008), Extended RANSAC Algorithm for Automatic Detection of Building Roof Planes from LiDAR DATA, Photogrammetric Journal of Finland, 21(1), pp. 97-109.

Thrun, S., Martin, C., Liu, Y., Hahnel, D., EmeryMontemerlo, R., Chakrabarti, D. \& Burgard, W. (2004), A Real-time Expectation-maximization Algorithm for Acquiring Multi-planar Maps of Indoor Environments with Mobile Robots, Robotics, IEEE Transactions on, 20(3), pp. 433-443.

Torr, P. H. \& Zisserman, A. (2000), MLESAC: A New Robust Estimator with Application to Estimating Image Geometry, Computer Vision and Image Understanding, 78(1), pp. 138-156.

Triebel, R., Burgard, W. \& Dellaert, F. (2005), Using Hierarchical EM to Extract Planes from 3D Range Scans, Proceedings of the 2005 IEEE International Conference on Robotics and Automation, pp. 4437-4442.

Truong-Hong, L., Laefer D. F., Hinks, T. \& Carr, H. (2013), Combining an Angle Criterion with Voxelization and the Flying Voxel Method in Reconstructing Building Models from LiDAR Data, Computer-Aided Civil and Infrastructure Engineering, 28(2), pp. 112-129.

Vidal R., Ma Y. \& Sastry, S. (2005), Generalized Principal Component Analysis (GPCA), Pattern Analysis and Machine Intelligence, IEEE Transactions on, 27(12), pp. 1-15.

Vidal, R. (2011), Subspace Clustering, IEEE Signal Processing Magazine, 28(2), pp. 52-68.

Vosselman, G. (2009), Advanced Point Cloud Processing. Photogrammetric Week, vol. 9, pp. 137-146.

Walsh, S. B., Borello, D. J., Guldur, B., \& Hajjar, J. F. (2013). Data Processing of Point Clouds for Object 
Detection for Structural Engineering Applications. Computer Aided Civil and Infrastructure Engineering, 28(7), pp. 495-508.

Zhang, C. and Elaksher, A. (2012), "An Unmanned Aerial Vehicle-Based Imaging System for 3D Measurement of Unpaved Road Surface Distresses," Computer-Aided Civil and Infrastructure Engineering, 27(2), pp. 118-129.

Zhang, G., Karasev, P., Brilakis, I., \& Vela, P. A. (2012), A Sparsity-inducing Optimization Algorithm for the Extraction of Planar Structures in Noisy Point-cloud Data. Proceedings of ASCE International Workshop on Computing in Civil Engineering, pp. 317-324.

Zhang, T., Szlam, A. \& Lerman, G. (2009), Median Kflats for Hybrid Linear Modeling with Many Outliers, Proceedings of Workshop of Subspace Methods, pp. 234241.

Zhang, T., Szlam, A., Wang, Y. \& Lerman, G. (2010), Hybrid Linear Modeling via Local Best-fit Flats, Proceedings of the IEEE Conference on Computer Vision and Pattern Recognition, pp. 1927-1934. 